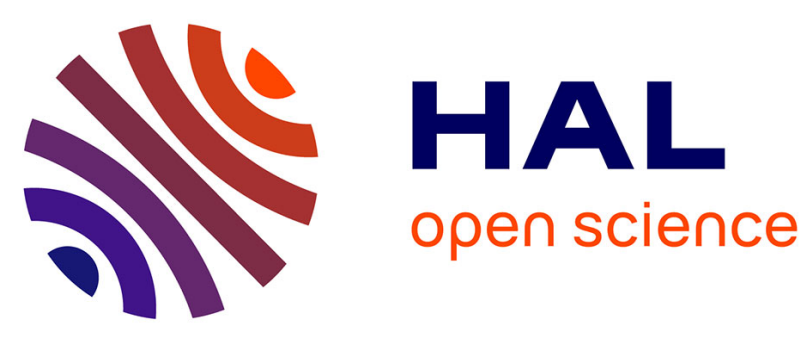

\title{
La psychanalyse et l'islam
}

Fethi Benslama

\section{To cite this version:}

Fethi Benslama. La psychanalyse et l'islam . Revue de l'enfance et de l'adolescence RAFEF-GRAPE, 2005, Jalousie et envie 4 (62 ), pp.91- 100. 10.3917/lett.062.100 . hal-01502417

\section{HAL Id: hal-01502417 https://hal.science/hal-01502417}

Submitted on 5 Apr 2017

HAL is a multi-disciplinary open access archive for the deposit and dissemination of scientific research documents, whether they are published or not. The documents may come from teaching and research institutions in France or abroad, or from public or private research centers.
L'archive ouverte pluridisciplinaire HAL, est destinée au dépôt et à la diffusion de documents scientifiques de niveau recherche, publiés ou non, émanant des établissements d'enseignement et de recherche français ou étrangers, des laboratoires publics ou privés. 


\section{LA PSYCHANALYSE ET L'ISLAM}

Fethi Ben Slama

ERES | « La lettre de l'enfance et de l'adolescence »

$2005 / 4 n^{0} 62 \mid$ pages 91 à 100

ISSN 2101-6046

ISBN 2749204429

Article disponible en ligne à l'adresse :

http://www.cairn.info/revue-lettre-de-l-enfance-et-de-ladolescence-2005-4-page-91.htm

\section{Pour citer cet article :}

Fethi Ben Slama, «La psychanalyse et l'islam », La lettre de l'enfance et de l'adolescence 2005/4 ( $\left.\mathrm{n}^{\circ} 62\right)$, p. 91-100.

DOI 10.3917/lett.062.100

Distribution électronique Cairn.info pour ERES.

(C) ERES. Tous droits réservés pour tous pays.

La reproduction ou représentation de cet article, notamment par photocopie, n'est autorisée que dans les limites des conditions générales d'utilisation du site ou, le cas échéant, des conditions générales de la licence souscrite par votre établissement. Toute autre reproduction ou représentation, en tout ou partie, sous quelque forme et de quelque manière que ce soit, est interdite sauf accord préalable et écrit de l'éditeur, en dehors des cas prévus par la législation en vigueur en France. Il est précisé que son stockage dans une base de données est également interdit. 


\section{Fethi Benslama \\ La psychanalyse et l'islam .}

En quoi l'islam est-il concerné par la psychanalyse?

D’une manière générale, le développement de la psychanalyse est étroitement lié à la fois au monothéisme et à l'émergence d'un monde fondé sur la science. Pour qu'elle ait droit de cité dans un pays, il faut qu'un certain nombre de conditions soient remplies. La première, fondamentale, est qu' il faut que l'approche de la folie devienne scientifique, grâce à la mise en place dans le système de santé d'un secteur psychiatrique, sur lequel viendra se greffer la psychanalyse. Or, si l'on regarde le monde musulman, on s'aperçoit que, sauf dans quelques pays très modernisés, le traitement de la folie et de la souffrance psychique reste soumis à des théories et à des pratiques fondées sur la démonologie, l'animisme...

\section{... Et la religion?}

Oui, mais ce n'est peut-être pas le plus important dans la mesure où la religion elle même peut subir l'influence de la science. En France, par exemple, l'attrait pour la psychanalyse, au début du siècle dernier, n’a pas été limité par le fait que les sujets étaient encore marqués par l'empreinte religieuse.

La deuxième condition a trait à l'existence d'un État de droit et d'un environnement démocratique. Car l'exercice de la psychanalyse suppose qu'on accepte un certain type de parole, de liberté avec la parole. Il faut que ce que quelqu'un va dire dans le lieu où se déroule une analyse, suivant le principe de l'association libre, ne puisse en aucun cas être utilisé contre lui à des fins policières, dans le

Fehti Benslama, psychanalyste, professeur de psychopathologie, Université Paris VII, auteur de La psychanalyse au risque de l'islam, Paris, Aubier, 2002.

* Le texte intégral de cet entretien réalisé par Hamid Barrada et Renaud de Rochebrune a été publié par J.A./L'intelligent N²327-2328, du 14 au 27 août 2005. 
cadre d'un quelconque système de répression. Il faut que l'État de droit sauvegarde et garantisse un tant soit peu la possibilité pour un patient de tout dire, mais aussi, pour des institutions psychanalytiques de travailler [...].

Un troisième facteur limite l'essor de la psychanalyse en terre d'islam : le discours très répandu tendant à présenter la psychanalyse comme une "science juive ». Il faut dire que la psychanalyse est l'une des rares disciplines, peut-être la seule, à avoir été inventée par un seul homme. Mais Sigmund Freud, même s'il ne reniait pas ses liens avec le judaïsme, n'était pas du tout religieux et se proclamait même athée. Dès les années 1920, il a pris des positions extrêmement courageuses. À un groupe de juifs qui lui demandait de soutenir le sionisme, il a répondu que ceux qui allaient s'installer en Palestine allaient immanquablement provoquer des troubles avec les Arabes, qu'ils n'avaient aucune raison d'aller là-bas.

On reproche encore à la psychanalyse son athéisme. Mais Freud, en tant que psychanalyste, cultivait ce que l'on pourrait appeler un athéisme suppositionnel : pour travailler, pour penser, on suppose que Dieu ne se mêle pas de tout. Ce qui était d'ailleurs, à l'époque, la position de nombreux savants, y compris musulmans, qui ne faisaient pas intervenir Dieu dans leur recherche.

Et, enfin, l'université, pourtant ouverte à l'enseignement et à la diffusion de beaucoup d'autres sciences humaines, n'a pas aidé à faire évoluer les choses dans les pays musulmans.

Cette difficulté de la psychanalyse à pénétrer en terre d'islam n' a-t-elle pas aussi des raisons plus théoriques? Ne dit-on pas que le complexe d'Edipe, qui est au cour de la théorie freudienne, n'est pas universel...

L'inanité de ce genre d'affirmation a été démontrée. Nous avons eu suffisamment de musulmans sur nos divans pour constater qu'ils ne sont pas différents des autres patients, qu'ils n'affrontent pas une problématique œdipienne différente. Il n'y a donc pas d'obstacle théorique, ou même simplement technique, pour analyser des musulmans. Sauf peut-être le cas de sujets très religieux, très croyants...

Justement : est-il possible pour des patients d'admettre le surgissement de phénomènes inconscients quand ils sont très croyants et qu'ils entendent tout attribuer, à commencer par leurs symptômes, à la volonté de Dieu ? Comment accepter les postulats de la psychanalyse si on pense que tout se ramène à "Inch Allah!"?

Avec l'expérience de ma pratique, je peux vous dire que quand des gens viennent vous voir avec une demande sincère, avec une vraie souffrance psychique, il y a souvent au début, notamment avec les jeunes, des interrogations sur la question religieuse $[. .$.$] . C'est le travail de l'analyste, de tout faire pour éviter qu'on$ aborde frontalement ce qui risque de créer de la résistance à l'analyse, d'enfermer le patient dans une voie sans issue. Quand le sujet a pu faire un pas de côté, il se préoccupe vite d'autre chose. Et ce n'est qu'ensuite que la question reviendra, mais alors elle aura pu être réélaborée : le sujet, ayant acquis une certaine liberté psychique, une certaine mobilité psychique, pourra aborder les mêmes problèmes avec un sens critique. Il s'apercevra par exemple que le fondement de la religion, et ce n'est pas vrai que pour l'islam, c'est la culpabilité. Et que cette culpabilité est, dans sa vie, une entrave considérable $[. .$.$] .$ 
Face à la psychanalyse, il n'y a donc aucune spécificité concernant les musulmans?

Il faudrait d'abord s'entendre sur le terme. De quels musulmans parle-t-on? Je prétends que le musulman en général n'existe pas. Il y a des musulmans, très différents les uns des autres. Ne serait-ce que parce qu'ils appartiennent à des temps historiques différents. Il y a le musulman qui appartient au monde traditionnel, qui n'a pas perdu ses repères et entend les conserver. Il y a le musulman pour lequel la tradition ne suffit pas et qui veut en revenir à quelque chose de plus fondamental, selon la voie des salafistes. Il y a le musulman qui accepte la modernité sans rejeter la tradition et qui adopte une position de compromis. Et il y a ceux qui continuent à se dire musulmans mais qui n'ont plus que des traces de foi $[\ldots]$.

Tous les Arabes se réferent pourtant à la même langue. Or l'on sait que la psychanalyse accorde une importance majeure au rapport du sujet à la langue...

La grande différence réside effectivement dans les langues, et même dans les idiomes - l'arabe de l'égyptien n'étant pas, bien sûr celui qu'on parle au Maroc. Car c'est par la langue maternelle que passent tous les processus de traduction consciente et inconsciente des événements de la vie psychique. Pour la psychanalyse, l'important, est cependant moins la langue qu'on parle que celle au travers de laquelle " on a été parlé »; c'est-à-dire la langue dans laquelle on a été élevé dans son jeune âge, dans laquelle on a reçu les premiers soins, dans laquelle, même avant notre naissance, nous avons existé en étant déjà nommés. Le sujet humain est « parlé » avant même de parler lui-même. Et quand il se met à le faire, c'est à partir de cet immense discours déjà tenu à son propos. Et d'une langue qui structure son univers psychique [...] Les signifiants d'une langue ont des articulations, des cohérences et c'est cela l'essentiel dans la formation du psychisme.

Avez-vous besoin de repasser par la langue arabe pour travailler avec certains patients, pour pouvoir interpréter certains de leurs propos, même quand la cure est menée en français?

Bien entendu. Mais ma clientèle étant internationale, cela ne joue pas que pour l'arabe. Quand je ne connais pas la langue maternelle d'un patient, il me parait parfois important qu'il m'explique l'étymologie d'un mot ou le sens d'une expression. Il se passe dans une cure des choses très étranges qui donnent l'impression d'une sorte de traduction d'inconscient à inconscient. Un jour, une patiente algérienne qui n'avait pas l'arabe pour langue maternelle, mais le berbère, que je ne comprends pas, me parle en français d'un rêve ou plutôt d'un cauchemar. Sans entrer dans le détail, elle voyait, en sortant de chez elle, un lapin dans le jardin. Moi, faisant mon travail d'analyste, je fais une association et ce lapin, je ne sais pourquoi, m'évoque un fotus. Je lui demande alors comment on dit lapin en berbère. Elle répond. Je lui demande alors si le mot qu'elle vient de prononcer a un autre sens et elle me dit qu'il signifie aussi... foetus.

[...] 


\section{Un musulman sur un divan, dites-vous, cela n'a rien de spécifique. Mais un émi- gré?}

Un émigré, certainement. Même quand cette émigration est ancienne ou n'a pas été vécue directement. On est là dans une problématique de la rupture, avec sa langue, les siens, son pays d'origine. Et cette rupture, cette confrontation avec la dimension de l'exil, a des conséquences. Pour certains, elle produit un effet traumatique : ils passeront une bonne partie de leur vie à réagir en fonction de ce traumatisme, à l' « élaborer » comme disent les psychanalystes. Pour d'autres, ce n'est pas si grave, même si cela reste important. Certains vont vouloir à tout prix agir, entreprendre. Ils éprouveront un désir extraordinaire de trouver une place dans la société où ils vivent. D'autres, en revanche, vivront l'exil comme un obstacle qui les empêche de faire quoi que ce soit. Souvent, si cela se passe mal, c'est parce que cette rupture vécue directement par les parents n'a pas été élaborée par la génération précédente, voire celle d'avant : on a fait comme si elle n'avait pas eu lieu. Il arrive que des enfants de migrants se trouvent devant des parents qui ne leur ont jamais parlé de leur pays d'origine, qui sont restés muets sur les raisons et la souffrance de l'exil. D'autant que cet exil peut avoir été vécu à la fois comme la réalisation d'une sorte de rêve - celui de partir ailleurs - et comme un acte accompagné d'une immense culpabilité, comme s'il s'agissait d'une transgression, voire d'une trahison. Or ces sentiments sont transmis aux enfants à leur insu.

\section{Ce qui est grave, c'est donc de refouler tout ce qui concerne l'exil?}

Dans ces cas difficiles il n'est pas seulement question de refoulement mais de traumatisme, ce qui est plus grave. Selon la théorie de Freud, on ne se dit jamais qu'on a subi un traumatisme au moment où il a lieu. Ce n'est que dans l'aprèscoup, quand quelque chose vient rappeler l'événement traumatique, qu'on s'aperçoit qu'on l'a subi. Apparaît alors ce qu'on appelle "le retour du refoulé »: on s'aperçoit que le fait traumatique lui-même avait été refoulé. Et à ce moment-là il devient possible de réélaborer son histoire. Mais il y a une autre théorie du traumatisme, celle d'un disciple de Freud, Sandor Ferenczi. Elle évoque le rejet d'une partie de soi-même qui accompagnerait le traumatisme. Et ce qu'on remarque chez les migrants qui souffrent le plus semble corroborer cette seconde théorie.

Il y a dans Mémoires d'immigrés, le célèbre documentaire de Yasmina Benguigui, une scène qui illustre ce phénomène. Un Maghrébin quitte son pays en bateau et, au moment où celui-ci prend le large, il jette sa chechia dans la mer. Un geste fréquent, avec des variantes, quand on part loin de son lieu d'origine et qui semble traduire une propension au rejet d'une partie de soi-même. Le plus souvent, ceux qui ont vécu cette sorte d'automutilation ne s'en souviennent pas. Ils se savent diminués, mais ils ne savent pas pourquoi. Ils ne savent pas ce qu'ils ont rejeté d'eux-mêmes, et vont donc le rechercher en vain.

À la demande d'un juge, j'ai reçu un jour un Tunisien et son fils, Ali, qui avait agressé une femme dans la rue. Le père, qui travaillait en France depuis trente ans, m'a expliqué que l'adolescent avait été gravement et durablement perturbé par un événement survenu alors qu'il avait 7 ou 8 ans en Tunisie : un jour qu'il revenait de l'école, d'autres garçons avaient déchiré ses cahiers. N'ayant pas réussi à le faire soigner là-bas, il avait décidé de ramener son fils en France, avec sa mère, en laissant au pays ses autres enfants qui, majeurs, n'avaient pas droit au regroupement 
familial. Le fils ayant confirmé les dires de son père, je me suis dit que je reparlerai de cela avec lui, au cours d'un prochain entretien. Et puis, au moment de partir, le père me dit : "Vous voyez, Ali a déchiré notre famille. » Ce retour du thème du déchirement m’a évidemment frappé. «Mais monsieur, lui ai-je répondu, c’est vous qui avez décidé de partir, c’est vous qui avez déchiré votre famille. » Le père voyait dans son fils l'agent du déchirement alors qu'il en était seul responsable. Et voilà pourquoi Ali allait si mal : il portait cette souffrance familiale à la place de son père sans pouvoir le reconnaître, dans le silence et le mutisme. Du coup, après cette intervention, c'est le père qui s'est effondré et qui a commencé un traitement avec moi.

La situation est-elle semblable pour tous ceux qui connaissent l'exil, qu'ils viennent par exemple du nord ou du sud du Sahara?

Quand on travaille, sur la clinique de l'exil, on s'aperçoit que les différences culturelles ne jouent aucun rôle. Ce qui est important, c'est la façon dont un homme vit son déplacement, la façon dont il parvient, avec ses ressources psychiques, à surmonter les effets de la rupture, le déchirement dont nous venons de parler. Je m'inscris donc en faux contre les théories de ceux qui, comme Tobie Nathan, s'imaginent pouvoir soigner les gens avec leur culture. À tant faire, pourquoi ne pas aller chez les guérisseurs?

\section{La psychanalyse, disiez-vous, est inséparable du monothéisme...}

La psychanalyse, je le répète, est née avec le monde moderne, avec l'apparition du sujet moderne, du sujet de la science. Un sujet, autrement dit, qui se met à penser son existence à partir de la rationalité scientifique. Ce qui est très particulier puisque la science, en même temps, élimine la question du sujet : lorsqu'on est dans un univers scientifique, on ne prend pas en compte a priori la subjectivité. Mais Freud a replacé la question de la subjectivité au centre des préoccupations, en la traitant selon une démarche scientifique. Alors, pourquoi ce rapport au monothéisme ? Justement parce que là où la science apparaît, devient dominante, l'ancien sujet, qui était un sujet religieux, a été chassé, éliminé. La religion n'est plus désormais qu'un élément privé. Dieu ne gouverne plus le monde, il est, si l'on peut dire, désactivé. Il y a donc une sorte de déconnexion avec ce qui était à la base de la subjectivité. Tout ce qui concerne la religion va être, d'une certaine manière, rejeté. Même si cela reste actif de façon inconsciente.

Que tout cela se passe dans l'univers des religions monothéistes n'est cependant pas un hasard. d'une manière quelque peu paradoxale, on pourrait soutenir que les monothéismes sont naturellement ouverts à la science, voire même que la science y est déjà potentiellement présente. Le Dieu unique, en effet, est à la fois présent et absent : il n'appartient pas à la nature, il est inaccessible. Chez les musulmans et les juifs on ne peut même pas le nommer. Sa présence est immatérielle, abstraite. Et c'est cela qui pourrait avoir servi de modèle à ce fondement de la science qu'est l'abstraction, qui aurait permis l'avènement de la science, l'émergence d'un univers scientifique. Et de fait, c'est bien dans l'aire des trois religions monothéistes que, dans les temps modernes, on a le plus développé des savoirs et des discours scientifiques. Lesquels, pour exister, impliquent un athéisme suppositionnel. [...] 
Vous évoquiez un second apport de l'islam à la psychanalyse. Quel est-il?

J'ai envie, en m'appuyant sur l'islam, de questionner la psychanalyse à propos de problèmes supposés réglés. En particulier, je voudrais réexaminer cette affirmation de Freud selon laquelle Dieu serait le père. Je prends très au sérieux l'enseignement de l'islam, à ce sujet, c'est-à-dire que Dieu n'est pas le père. Je pense que, pour bien lire le Coran, il faut remonter en amont, jusqu'à la Bible. On s'aperçoit alors que, dans la Genèse, figure quelque chose d'essentiel à la généalogie du monothéisme. À savoir que Isaac n'est pas le fils d'Abraham, lequel n'est qu'un père symbolique puisque c'est Dieu qui doit intervenir dans le corps de Sarah pour qu'elle mette miraculeusement au monde cet enfant. Donc Isaac est le prototype de Jésus : c'est la même position. Tout comme Abraham est dans la même position que Joseph. Dans le judaïsme et dans le christianisme, Dieu intervient ainsi dans la procréation. Ce qui n'est pas le cas dans l'islam : Abraham ayant fécondé sa servante Agar, il est un père réel. Dieu n'intervient pas, il est créateur mais pas procréateur.

Il y a donc une bifurcation généalogique. D’un côté, celui de l'islam, Abraham est le père réel, Dieu n'intervient pas dans la procréation d'Ismaël, lequel est exclu et renvoyé dans le désert avec sa descendance. De l'autre, Dieu intervient dans la procréation d'Isaac et de Jésus. C'est à partir de là qu'apparaissent les deux lignées du monothéisme. Et qu'il devient possible d'examiner comment se constitue le mythe du père dans chaque civilisation.

Les musulmans n'ont pas besoin d'articuler la figure du père à Dieu. D'ailleurs le père n'est pas si bien vu dans le texte coranique, il est avant tout une source d'illusion. Ainsi l'islam apparaît donc comme une religion du fils, qui n'accorde aucun privilège au père dans sa construction dogmatique. Et si le patriarcat règne si souvent en terre d'islam, c'est pour des raisons antérieures à l'avènement de l'islam. Le mot père n'est d'ailleurs jamais utilisé au singulier dans le texte coranique, on parle toujours des pères, ou des pères et mères à la fois.

\section{Quelles conséquences pour la psychanalyse?}

Dans la mesure où la psychanalyse est fondée sur une théorie du père, elles sont évidemment importantes. Si on ne pense plus Dieu à partir du père mais à partir d'une autre place que celle du père, beaucoup de choses changent.

Pour Freud, Dieu n'est qu'une projection, une image du père, protecteur, etc. Pour Lacan, au contraire, on ne peut penser le père qu'à partir de Dieu, lequel occupe une place vide, la place de l'Autre. Mais il conserve le lien entre Dieu, le père et la paternité, reprenant d'ailleurs dans un de ses séminaires cette formule toute chrétienne des noms-du-Père. Les noms-du-Père sont des signifiants qui, dans le langage, sont essentiels à la construction du sujet. Mais si Dieu occupe vraiment une place vide, pour quelle raison devrait-on conserver cette notion?

Il faut savoir que, dans la psychanalyse, le père n'est pas le père géniteur, c'est une métaphore. Il appartient à l'univers symbolique. Si un homme se prend vraiment pour le père, c'est catastrophique, il rend ses enfants fous. Pour faire simple, disons qu'en se réinterrogeant sur le père, on s'interroge du même coup sur les fondements de l'univers symbolique, et donc sur les fondements de la psychanalyse elle-même. 
Dans votre dernier livre, vous évoquez la place de la femme et du féminin dans l'islam. S'agit-il d'un point crucial du rapport entre islam et psychanalyse?

C'est en tout cas central dans mon travail. J'ai écrit ce livre avec l'idée que, si je traitais des périodes de refoulement qui ont marqué l'histoire de l'islam, si je ramenais ces choses refoulées à la surface, je provoquerais peut-être des effets libérateurs. Freud pensait d'ailleurs que toutes les religions ont à voir avec le refoulement. Au fil de ma recherche, j'en suis arrivé à la conviction que la situation de la femme dans l'islam n'est pas ce qu'on en dit le plus souvent. On soutient que l'islam repose sur un système patriarcal, ce qui n'est pas très original. Mais on peut aussi, et surtout, soutenir que le cœur de ce système est le refoulement de la question féminine. C'est plus spécifique et cela méritait examen.

Une fois encore, je suis retourné à l'origine, à Abraham et à la Genèse. Et il m'est apparu que, dans le texte coranique, tous les protagonistes de la Genèse sont présents, même s'ils ne sont pas nommés. Tous sauf un : Agar, la servante de Sarah, qui est la femme d'Abraham, et la mère d'Ismaël, l' " ancêtre des Arabes ». Le geste de répudiation d'Agar accompli par Abraham à la demande de Sarah, après la naissance d'Ismaël, s'est donc poursuivi à travers par son effacement dans le texte coranique. Est-ce parce qu'il était difficile pour le Prophète de dire aux fiers Arabes du désert que leur mère ancestrale était une servante répudiée de surcroît ? De fait, il y a eu un débat entre les musulmans à ce sujet au VIII ${ }^{\mathrm{e}}$ siècle. Toujours est-il qu'Agar, la seule femme qui, d'après la Bible et comme l'a noté Spinoza, a vu Dieu et s'est vu promettre par Lui une descendance équivalente à celle d'Abraham, est absente du Coran. La figure de la mère fondatrice de l'islam, celle dont procède la généalogie et, par là même, l'ordre symbolique, ne figure donc pas dans le texte fondateur : voilà un vrai refoulement! Pour reconstituer le récit originaire, le coup de génie du Prophète fut alors d'imaginer des retrouvailles entre Abraham et Ismaël, une scène qui n'existe pas dans la Bible, tous deux entreprenant ensuite de construire la Maison de Dieu.

J'ai ensuite essayé de montrer que le fondateur de l'islam est dans une posture mariale, ce qui expliquerait d'ailleurs la place importante de Marie dans le texte coranique. Marie et le Prophète étaient l'un et l'autre "vierges de toute lettre »- illettrés donc -, disent les textes sacrés. Et puis, un ange vient à Marie pour lui insuffler le verbe... et le même ange vient à Mohammed pour qu'il reçoive la lettre. Enfin, je me suis intéressé à l'étymologie du mot qara’a qui signifie à la fois « lit » et « conçoit » : la réception de la lettre - autrement dit : la capacité à recevoir de Dieu le texte sacré - serait équivalente à une gestation, elle consisterait à se mettre dans une position féminine vis-à-vis de Dieu. Trois éléments - la virginité, la visite de l'ange et la réception de la lettre - mettent donc Mohammed dans une position mariale. Autrement dit, du point de vue de l'islam, le Prophète serait plutôt à la place de Marie que de Jésus.

Même si elle a été refoulée par la suite, il y a dans l'islam, à son origine, au lieu de sa fondation, une part féminine essentielle qui se trouve confortée par le rapport entre Mohammed et sa première épouse. Khadija joue en effet un rôle capital lors de la Révélation. Elle reconnaît en Mohammed le prophète, lui enseigne la distinction entre l'ange et le démon, etc. Tant que Khadija, la première musulmane, a été vivante, aucun élément défavorable aux femmes n'a été introduit dans le texte de la Révélation, dans le Coran. Ce n'est qu'après sa mort qu'on note un 
basculement chez le Prophète. J'interprète ce basculement comme un refoulement de sa constitution en tant que sujet prophétique, donc un refoulement de la position féminine qui a accompagné la réception de la lettre et du Verbe. À partir de ce moment, c'est le Mohammed de Médine qui domine, c'est-à-dire celui de la conquête, l'homme d'État, etc. Il y a deux Mohammed. Le premier est dans la position mariale. Le second, qui devient un chef, dans une position qu'un psychanalyste pourrait qualifier de phallique, en tout cas une position plus masculine. Laquelle implique le refoulement de la position précédente.

\section{Quelle a été la conséquence de ce refoulement?}

L'islam traite les femmes d'une manière très particulière. La place de ces dernières est en effet très différente dans les autres textes monothéistes. Ce qui laisse à penser que la condition féminine en terre d'islam est en partie déterminée par ce phénomène. En lançant le débat sur la question de la féminité dans l'islam en débat, en tentant de lever ce refoulement, je peux espérer produire certains effets, même si, bien sûr, ce travail doit être poursuivi. En se demandant, par exemple, s'il n'y aurait pas eu, à l'origine, une sorte de surestimation du féminin, qui expliquerait qu'on ait par la suite tenté de réduire drastiquement sa place. Jusqu'à faire de l'islam une sorte de monument contre-féminin si je puis dire.

Cette question de la féminité dans l'islam peut-elle être reliée au phénomène islamiste?

On voit bien que la question de la femme, de son corps, de son statut, est un thème central, voire LE thème central, pour tous les mouvements islamistes. Ces derniers savent très bien que si on touche à cela, tout leur édifice risque de s'effondrer.

Quelles en sont les conséquences pour la psychanalyse?

Disons, pour donner au moins un exemple, que cela permet sans doute d'approfondir la théorie lacanienne de la jouissance. Et en particulier, comme je le développe dans mon livre à partir des Mille et Une Nuits, celle de la jouissance féminine, tellement mystérieuse.

Pensez-vous que cette interrogation sur certains points théoriques à partir de l'islam, puisse contribuer à une sorte de refondation de la psychanalyse?

Je n'irai pas jusque là. J'essaie simplement d'introduire des interrogations de nature à faire débat. Qu'est-ce qu'on appelle " père " ? S'il ne s'agit que du résultat d'une opération langagière, comme le soutiennent certains psychanalystes, peut-on dire que les pères soient les seuls capables de " porter » le registre du symbolique, les seuls porteurs de métaphore et de langage ? Et pas les mères, donc la moitié de l'humanité ? Est-ce que les femmes peuvent transmettre ce qui a rapport à l'ordre symbolique, sans en passer par le père ? Dans l'islam, on pose Dieu et on pose le langage comme n'ayant pas affaire au genre, au masculin et au féminin, à la paternité, etc. Le Dieu de l'islam n'est ni père, ni homme, ni femme, mais un Dieu de la parole. C'est très intéressant, car le Dieu de la parole véhicule l'espoir de voir apparaître une nouvelle forme de laïcité liée à une nouvelle conception de 
l'ordre symbolique. Évidemment, je simplifie beaucoup des questions compliquées, mais on peut mesurer ici l'importance des enjeux.

Le rôle du complexe d'Édipe ne dépend-il pas de la conception que l'on se fait du père?

La théorie de l'CEdipe est une théorie de la genèse du sujet humain. Elle dit, pour simplifier une nouvelle fois, que tout être humain naît dans le conflit du désir entre un homme et une femme. Cela concerne tous les sujets, musulmans compris bien sûr. Ce que je conteste, c'est qu'on utilise cette théorie pour tenter d'expliquer la genèse d'une culture et les fondements d'une civilisation. L'axiome œdipien permet de comprendre l'émergence du sujet humain, mais lorsqu'il s'agit de la constitution des cultures, il s'agit d'autre chose. 
«J'allais affronter mon frère devant tous, et je n'en menais pas large. Gunnar, plus grand et plus fort que moi, pratiquait les armes depuis longtemps. J'avais pour ma part peu d'expérience, ne possédant qu'un titre de gloire, une certaine force au tir à l'arc.

Je ne décrirai pas ce combat contre mon frère, qui fut l'un des pires moments de ma vie. Gunnar, sans pitié, me pulvérisa. Mon épée mordeuse me sauta trois fois des mains, et je me retrouvai par deux fois les quatre fers en l'air. La lame de mon frère, recouverte comme la mienne d'étoffe épaisse, ne pouvait trancher ni piquer. Je m'en sortis donc avec des bleus au corps... et surtout à l'âme.

Car ma déconfiture avait été publique. Mon père, que j'admirais tant, à qui je rêvais de ressembler un jour, avait pu constater quel piètre guerrier j'étais. Mes joues brûlaient de honte, je me souviens. Et pour la première fois de ma vie, je détestai Gunnar.

Je trouvai cependant un peu de réconfort dans les yeux de certains spectateurs : ma mère, ma petite sœur et aussi le demi-troll Dizir. [...]

- Demain, je donnerai une leçon à mes fils, annonça mon père en se levant pour gagner sa chambre. Dizir et moi, nous vous montrerons comment tenir une épée.

Gunnar pâlit à ces mots, car notre père semblait ne faire aucune différence entre nous deux : entre le vainqueur et le vaincu. J'étais un peu vengé. » L'école des loisirs, Paris, 2005, p. 22-23. 